\title{
Possible APPlication OF HeTERogeneous Robot Group TO SEARCH AND LOCALIZE IONIZING RADIATION SOURCES
}

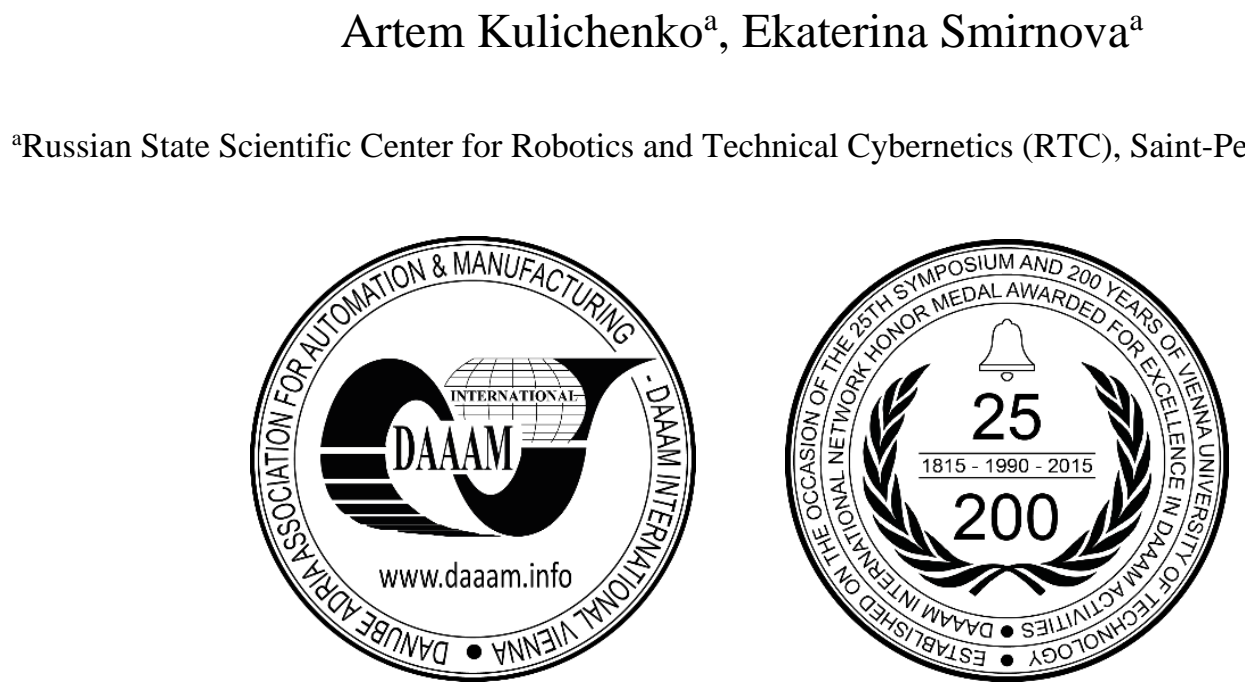

This Publication has to be referred as: Kulichenko, A[rtem] \& Smirnova, E[katerina] (2017). Possible Application of Heterogeneous Robot Group to Search and Localize Ionizing Radiation Sources, Proceedings of the 28th DAAAM International Symposium, pp.0859-0866, B. Katalinic (Ed.), Published by DAAAM International, ISBN 978-3-90273411-2, ISSN 1726-9679, Vienna, Austria

DOI: $10.2507 / 28$ th.daaam.proceedings. 120

\begin{abstract}
Let us define heterogeneous robot group as the main subject of research. The aim of this work is to investigate the possibilities when heterogeneous robot group can be used to search ionizing radiation sources. To compare the duration of operations, fulfilled by single robot and by robot group, experimental studies have been made. On the basis of the results, gained from the experiments, the strategy and procedures for ionizing radiation sources detection with given accuracy, are determined. Justification for efficiency increase is also given.
\end{abstract}

Keywords: Heterogeneous robot group; group robotics; complexes for radiation reconnaissance; UAV (unmanned aerial vehicle); group robots application possibilities

\section{Introduction}

Robots application appears to be the most appropriate for extreme environments, where both human work and activity are rather difficult, impossible or can be a real hazard for human life and health[1]. Such environments include working within ionizing radiation fields, for example, to mitigate consequences of technological disasters. The advantages of robot group application for the tasks of extreme robotics are quite obvious [2]. In general they are: greater range of operation that is due to the robots distribution within entire working area; extended operation abilities - that is due to mounting on each robot its individual actuator; and the higher success probability while fulfilling the task - that is due to the possibility of goals redistribution between the robots within group.

Let us consider the problem of robot group formation for the task of searching ionizing radiation source (IRS) and task planning for its work. IRS search and localization is a kind of very specific task, resulting in case of accidents or emergencies in IRS applying industries (of course, restricted IRS application is meant, for example in chemical industry, food industry, etc.), relating to operations and service of interim spent-fuel or industrial IRS storage facilities, or to the threats of terrorist attacks when the so-called "dirty bombs" can be used, and some other local accidents. 
On the one hand, such situations can be described as having relatively small and "known" accident zone. On the other hand, there can be no special team for consequences mitigation in the area of emergency, and this may lead to the necessity of delivering special equipment from local emergency centers nearby. Therefore, for the robot group the mobility is needed, and its transportation to the accident zone or emergency area should be carried out with cost-effective and relatively inexpensive means (vehicles) to also serve the base point for monitoring and control.

Let us define the optimal robot group configuration and the structure of robotic complexes (RC) needed for functional tasks accomplishment. To make a decision about robot group configuration for the tasks of IRS detection and localization, the following key considerations should be kept in mind:

- to organize data transmission between the group members in conditions of unstable communication as well as to significantly increase the communication range with ground-based robots, use of unmanned aerial vehicles (UAV) can provide best possibilities;

- to detect and define direction to IRS at the same time being at the safest possible source-distance, each member of the robot group should be equipped with special devices, providing remote sensing of the radiation field;

- to make it possible to solve localization and navigation tasks, members of the robot-group should also have some gridding equipment for geo-location (e.g. GPS/GLONASS). However, there should also be provided scenarios of performing operations within freak zones, when communication of ground-based robots with satellites can be lost.

\section{Group operation planning}

Factor of time is crucially important especially for the tasks of extreme robotics, often responsible for operation in zones/areas of heavy and rugged terrain.

Robot group operation planning should provide:

- reducing the time for operation accomplishment;

- saving energy due to optimal scheduling;

- making it possible to preliminary evaluate hazard level (e.g., level of radiation danger) and detail the maps for passability within area of operation with the help of UAV.

One more advantage of robot group application could be minimizing RC dose loads when working within the radiation fields. To achieve the advantages above, it is necessary to elaborate logic of functioning and develop typical scenario of robot group operation.

Despite the above mentioned, the following tasks should also be taken into consideration when designing the prototype:

- providing communication and interaction of members in the group;

- ensuring the group navigation using all available navigational information in general;

- creating the point of centralized management overall group control with corresponding hardware and software.

However, these general tasks are not covered by the subject area of this paper, focusing on the tasks of IRS search and localization, that can be solved on the basis of well-known theoretical approaches [2].

The paper describes and analyzes existing approaches and techniques of solving the tasks for IRS search and localization. On the basis of analysis given, the possibility of improving is stated - to be used for heterogeneous robot group operation tasks.

Technologies and devices of IRS search and localization are generally based on analyzing parameters of radiation field, that can be measured remotely, at IRS safe distant. For the task of IRS detection, gamma-ray direction finders are widely used. They are able of measuring course angle on target (that is ionizing radiation source) with the accuracy of 13 degrees [3].

While operating, the ground-based robot sequentially fulfils the tasks of:

- IRS detection;

- IRS localization in robot system of coordinates;

- IRS reaching for the robot to be capable of fulfilling operations with manipulator equipped.

\section{General description of the algorithm for IRS detection and localization by ground-based robot}

First and foremost, to start procedure of IRS detection, measurement of the intensity of the background radiation is performed. Then the detecting procedure begins: the mobile RC studies the area moving across and making stops from time to time. While searching, IRS location in relation to RC is considered to have two parameters: course angle to target a and distance $\mathbf{d}$ (see Figure 1). 


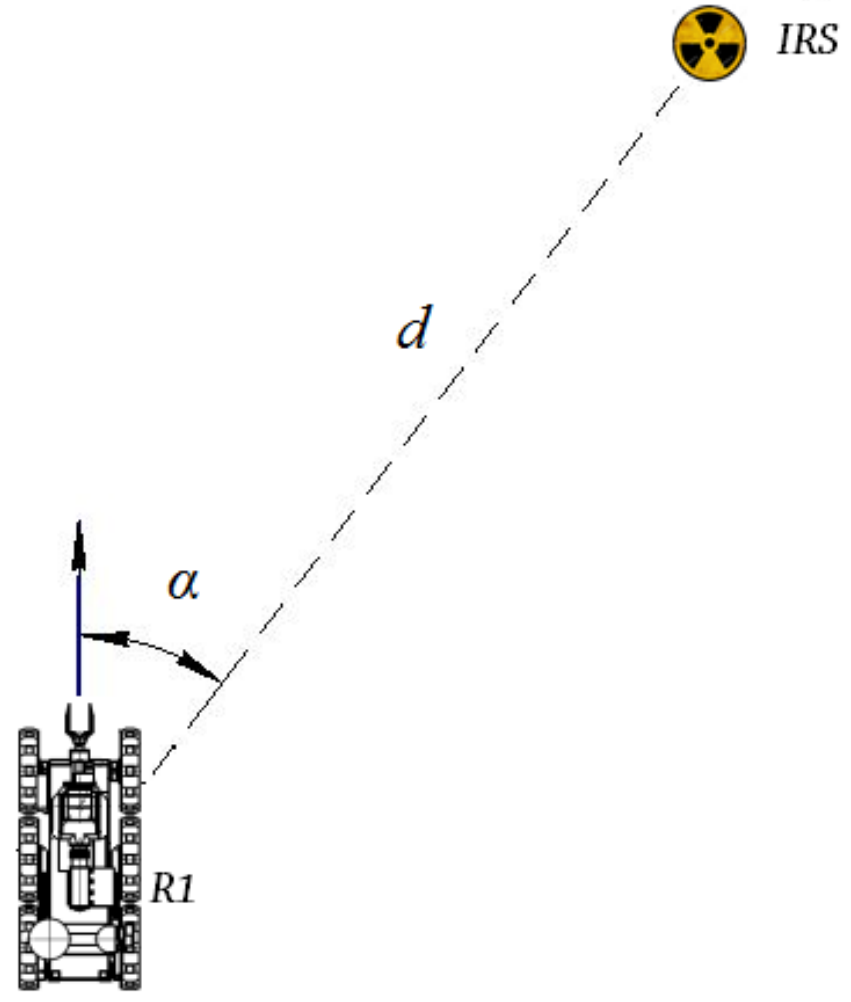

Fig.1. Finding Direction to IRS by the Robot (R)

Main measurements and statistical analysis based on their results for the robot to find IRS location, are performed during the stops. The procedures at points of stop suppose the following:

- within exposure period the robot registers the radiation via gamma-finder, estimates direction to the IRS and calculates direction estimation error;

- in case the robot has fulfilled controlled transfer (that is measuring the path covered) from the previous stop to the current one, the distance between RC and IRS is estimated by means of comparing radiation level at these two stops, and so the distance estimation error is calculated.

Taking into account the angle measured, RC then makes a turn in IRS direction and moves to it - with regard to measured distance. While moving, gradient of radiation level is controlled, and approximate estimation of distance to the IRS is calculated.

It is possible for the ground-based RC to define IRS location with accuracy enough to take IRS and put it into container with the help of manipulator. The main disadvantage of technology described is the following: when accomplished by the only (single) ground-based RC, the task of IRS localization cannot be solved with needed accuracy until the robot comes closer to the IRS location, e.g. enters higher radiation fields. The farther is IRS location, the weaker is radiation field intensity and the more is estimation error of distance $\boldsymbol{d}$, since radiation field gradient value is used for this very parameter evaluation. The closer is IRS location, the more is either risk of RC electronic components destruction or necessity of applying shielding alloys and radiation resistant elements, what makes the robot much more expensive. Improving accuracy of distance-parameter estimation is possible through triangulation method use, that is due to concurrent operation of the two ground-based RCs in our case.

\section{Simulation of the task-mission for a single RC. Selection of the robot group}

Due to statistical modeling program, it has become possible to simulate set of experiments for single robot to detect and localize IRS. The goal of simulation has united two main tasks: the first one - to calculate IRS average search time and the second one - to estimate the percentage of successful operations fulfilled by single RC in terms of minimal radiation exposure for both robot and RC operator. In accordance with the radiation safety standards, the exposure dose rate (EDR) of $50 \mathrm{mcR} / \mathrm{h}$ has been taken as minimal at the start point, and EDR of $250 \mathrm{mcR} / \mathrm{h}$ - as maximal acceptable exposure limit. The distance of 500 meter is considered as distance to the radiation source, that is standard radius for light-class robot operation zone. The value of radiation background is accepted as equal to $14 \mathrm{mcR} / \mathrm{h}$ (natural background, $\mathrm{NB}$ ) and to $28 \mathrm{mcR} / \mathrm{h}$ (natural increased background, NIB).

Based on simulation results, there is SD chart obtained, where we can see that angle estimation at robot starting point depends on exposure period. Please, see the results of simulation in Table 1. SD angle dependency graph on single element searching time within each simulation held is presented in Figure 2. 


\begin{tabular}{|c|c|c|c|}
\hline \multicolumn{4}{|c|}{ EDR at starting point, $\mathrm{mcR} / \mathrm{h}$} \\
\hline \multicolumn{4}{|c|}{50} \\
\hline \multicolumn{2}{|c|}{ NB } & \multicolumn{2}{|c|}{ NIB } \\
\hline \multicolumn{2}{|c|}{ initial conditions, option I } & \multicolumn{2}{|c|}{ initial conditions, option II } \\
\hline $\begin{array}{l}\text { Average mean } \\
\text { time of } \\
\text { exposure,s }\end{array}$ & RCS angle, $^{\circ}$ & $\begin{array}{c}\text { Average mean } \\
\text { time of } \\
\text { exposure,s }\end{array}$ & SD angle, ${ }^{\circ}$ \\
\hline 5989,74 & 0 & 28156,7 & 0,86 \\
\hline 3070,62 & 2,22 & 6481,61 & 2,22 \\
\hline 1148,63 & 4,19 & 2131,46 & 4,59 \\
\hline 345,79 & 6,06 & 608,28 & 6,82 \\
\hline 174,56 & 7,7 & 316,36 & 9,64 \\
\hline 134,42 & 8,8 & 242,94 & 9,9 \\
\hline \multicolumn{4}{|c|}{ EDR at starting point, $\mathrm{mcR} / \mathrm{h}$} \\
\hline \multicolumn{4}{|c|}{250} \\
\hline \multicolumn{2}{|c|}{ NB } & \multicolumn{2}{|c|}{ NIB } \\
\hline \multicolumn{2}{|c|}{ initial conditions, option III } & \multicolumn{2}{|c|}{ initial conditions, option IV } \\
\hline $\begin{array}{l}\text { Average mean } \\
\text { time of stop,s }\end{array}$ & $\underset{\circ}{S D \text { angle, }}$ & $\begin{array}{c}\text { Average mean } \\
\text { time of stop,s }\end{array}$ & $\underset{\circ}{\text { SD angle, }}$ \\
\hline 1841,94 & 0,86 & 2267,49 & 0,83 \\
\hline 416,67 & 2,2 & 499,51 & 2,31 \\
\hline 152,34 & 3,94 & 186,09 & 4,02 \\
\hline 46,1 & 5,98 & 54,06 & 6,7 \\
\hline 27,34 & 7,01 & 29,07 & 7,89 \\
\hline 22,03 & 7,9 & 24,08 & 8,5 \\
\hline
\end{tabular}

Table 1. Simulation results for SD dependencies of IRS angle estimation on exposure time at starting point
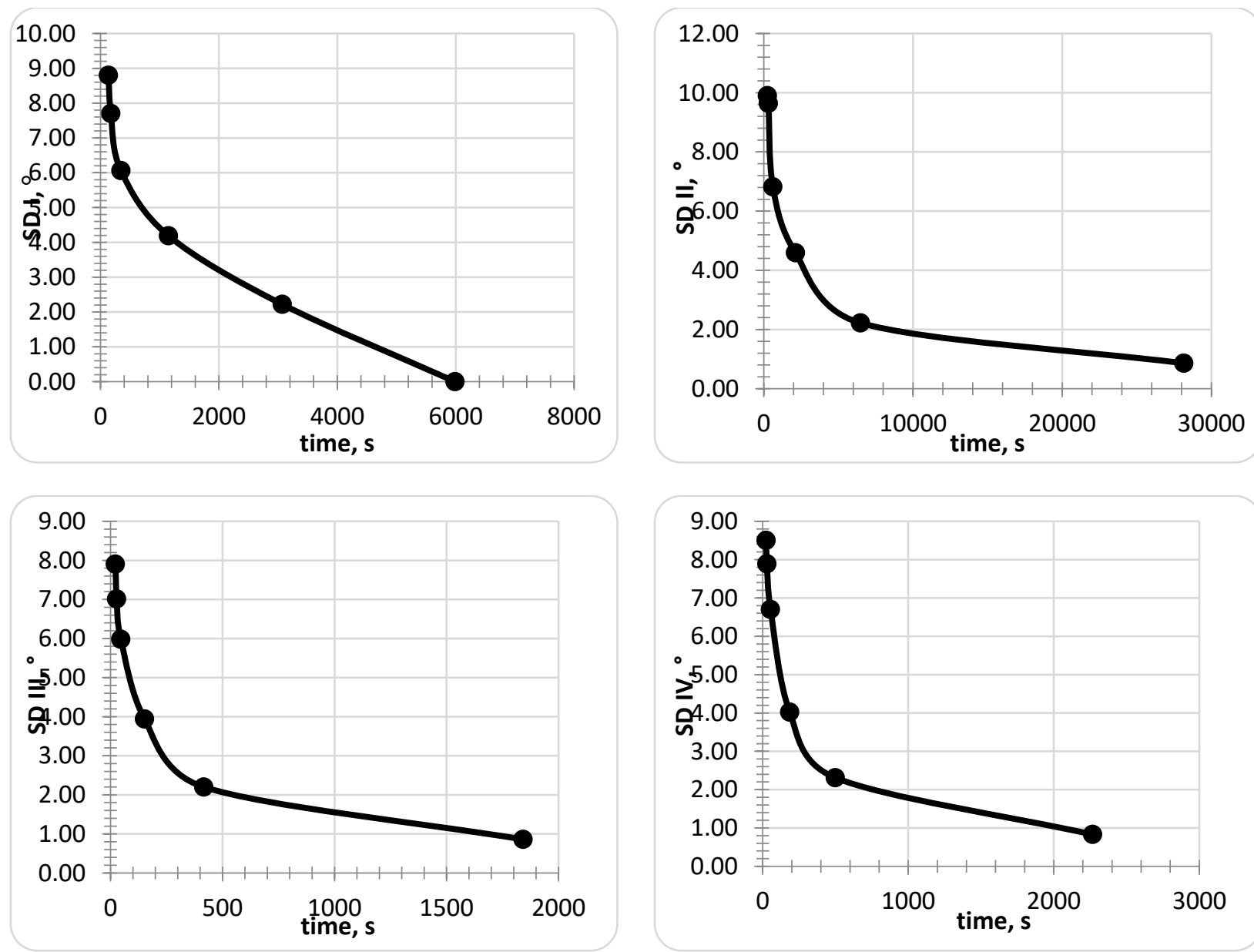

Fig. 2. SD dependencies graphs of IRS angle estimation on exposure time at starting point 
The data show that when starting from the safe zone (calculation options I and II), errors under $6^{\circ}$ are provided by increasing the duration of the first stop.

Then search-mission simulation has been made, that is - simulating automatic movements of the robot to IRS-source for each initial conditions option I-IV provided that the time of stop is 50 minutes. The search missions have been considered successful in case the robot reaches IRS location.

Simulation results have allowed to calculate the percentage of successful operations and average time for mission operation. When determining the average operation time, all missions have been taken into consideration, including the failed ones. Please, see simulation results presented in Table 2.

\begin{tabular}{|l|c|c|c|c|}
\hline \multirow{2}{*}{ Characteristics } & \multicolumn{4}{|c|}{ Value } \\
\cline { 2 - 5 } & \multicolumn{3}{|c|}{ Option } \\
\cline { 2 - 5 } & I & II & III & IV \\
\hline $\begin{array}{l}\text { Average mission operation time for } \\
\text { IRS searching, s }\end{array}$ & 3611,5 & 12291,33 & 4159,5 & 4074 \\
\hline Successful missions percentage, \% & 40 & 30 & 90 & 90 \\
\hline
\end{tabular}

Table 2. Simulation Results for IRS search-mission operation by single robot in the automatic mode

The simulations have shown that time of mission-operation for single robot takes up to $3.5 \mathrm{~h}$ at average, that is nearly the limit of operation-time for light-class robot without recharging or replacing the batteries. In terms of minimum radiation burden for the operator at the starting point, it is impossible to fulfill the task successfully at $100 \%$.

To reduce time-period for searching without increasing the radiation hazard for the operator becomes possible in case of coordinated IRS direction-finding at maximum possible safe distance by means of two and more RCs.

Equipped with device of radiation search, UAV is able to carry on radiation reconnaissance from the height, without being exposed. UAV application gives possibility for a number of ground-based robots to stay in relatively safe zone at the same time defining approximately the area of IRS location, to estimate possible obstacles within RCs operation zone, to provide reliable communication as well as to keep observation in the process of mission. However, when doing aerial radiation reconnaissance, there is one main technological disadvantage-significant IRS localization error due to the field spatial smoothing.

Thus the best result can be achieved when using the group of robots, consisting of:

- Ultra-light class ground-based RC equipped with gamma finder;

- Light-class ground-based RC equipped with gamma-finder, manipulator and container;

- Two UAVs of the helicopter type: one - for preliminary radiation reconnaissance, the other - for video surveillance tracking the progress of ground-based RCs operation and communication with them.

\section{Simulation of the task-mission for robot group. Results and analysis}

Let us analyze the IRS search-mission operation for two ground-based robots with UAV application. We assume that UAV use has allowed to define the following:

- border of the safe zone; and

- approximate area of IRS localization taking in mind the error of \pm 75 meters (due to field spatial smoothing and dynamics of UAV);

At the same time the following reasonable assumptions can be accepted:

- Placing the ground-based robots at the border of the safe zone is initially performed with the help of vehicle;

- On-Board GPS/GLONASS receiver system defines the coordinates of robots within absolute coordinate system with negligible error;

- Robots are identically oriented in azimuth.

First and foremost it is necessary to find the best place for starting points of the robots in correspondence to each other, having in mind, that the best ones are such initial positions where mission successful operation probability is equal to $100 \%$ and the time spent for the mission-task is the least. You can find the task illustrated in Figure 3, where: a prior preliminary IRS location is for IRS point, and the initial starting positions of robots are shown as R1 and R2. 


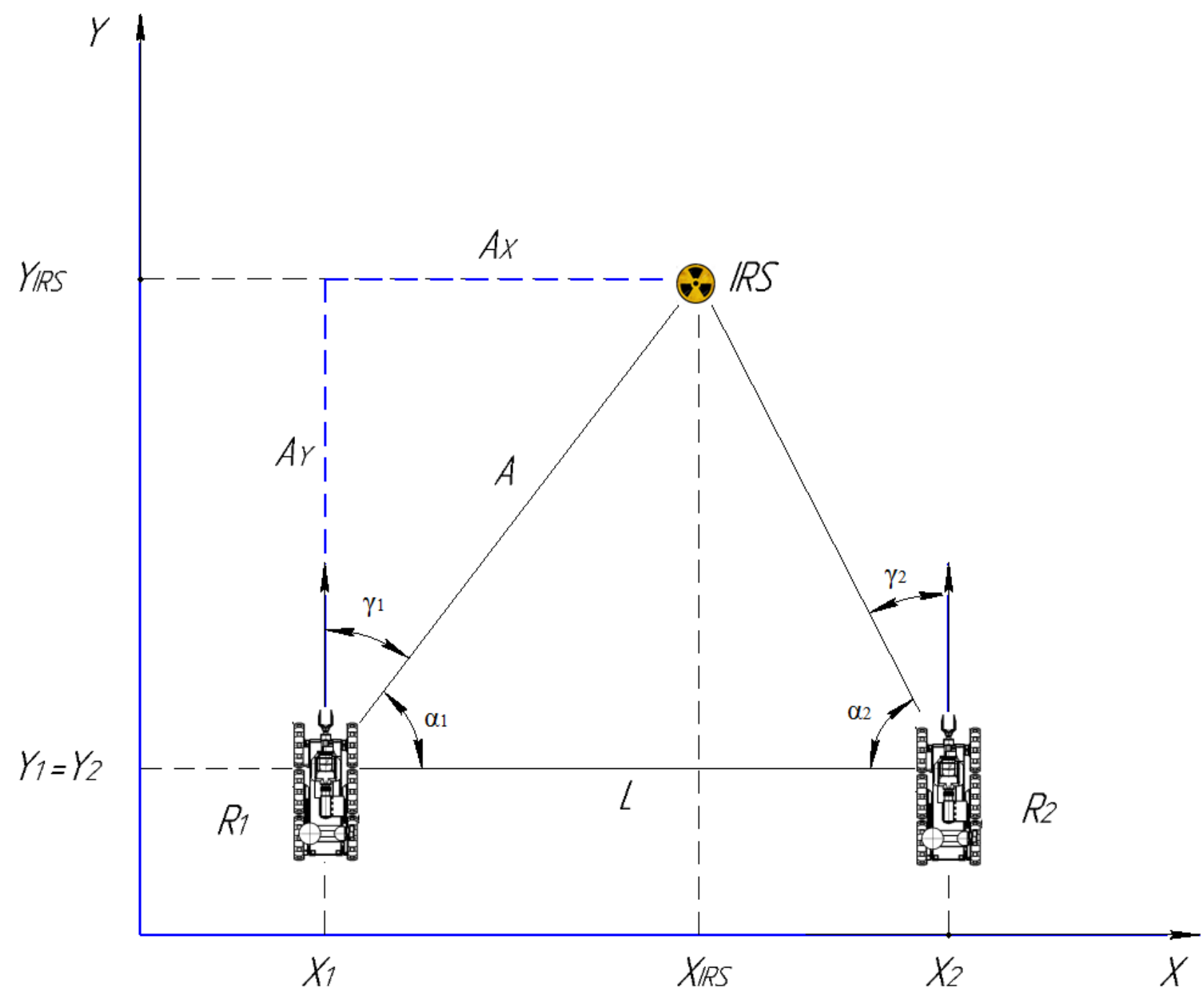

Fig. 3. Example of two robots positioning in the starting points at the beginning of IRS search-mission

It is quite obvious, that the coordinates of this source $\mathrm{X}_{\text {IRS }}$ и $\mathrm{Y}_{\text {IRS }}$ can be found from the system of equations:

$$
\left\{\begin{array}{l}
X_{I R S}=X_{1}+L \frac{\cos \left(\gamma_{2}\right)}{\sin \left(\gamma_{1}+\gamma_{2}\right)} \cdot \sin \left(\gamma_{1}\right) \\
Y_{I R S}=Y_{1}+L \frac{\cos \left(\gamma_{2}\right)}{\sin \left(\gamma_{1}+\gamma_{2}\right)} \times \cos \left(\gamma_{1}\right)
\end{array}\right.
$$

Values $X_{\text {IRS }}$ and $Y_{\text {IRS }}$ do depend on uncorrelated random variables - estimates of the angles $\gamma_{1}$ and $\gamma_{2}$, and therefore are also random values. To calculate SD estimates of these variables, it is necessary to find their dispersions using method of linearization [4]:

$$
\left\{\begin{array}{l}
\sigma_{X_{I R S}}^{2}=\sum_{i=1}^{n}\left(\frac{\partial X_{I R S}}{\partial \gamma_{i}}\right)^{2} \cdot \sigma_{\gamma_{i}}^{2} ; \\
\sigma_{Y_{I R S}}^{2}=\sum_{i=1}^{n}\left(\frac{\partial Y_{I R S}}{\partial \gamma_{i}}\right)^{2} \cdot \sigma_{\gamma_{i} ;}^{2} ;
\end{array}\right.
$$

where $б \gamma_{1}$ и $б \gamma_{2}$ - are SD angles estimates for IRS direction, $\gamma_{1}$ and $\gamma_{2}$ correspondingly. Setting the range of errors for the coordinates determining, restrictions on the values $б \gamma_{1}$ и $б \gamma_{2}$ can be got.

Figure 4 shows a graphical interpretation of $\sigma \gamma_{1}$ and $\sigma \gamma_{2}$ correlation and failures of IRS finding. Rhombus (blue line) defines the boundaries of IRS localization area, and the projection of rhombus diagonals are $3 \cdot 6 X_{\text {IRS }}$ and $3 \cdot 6 Y_{\text {IRS }}$ correspondingly. 


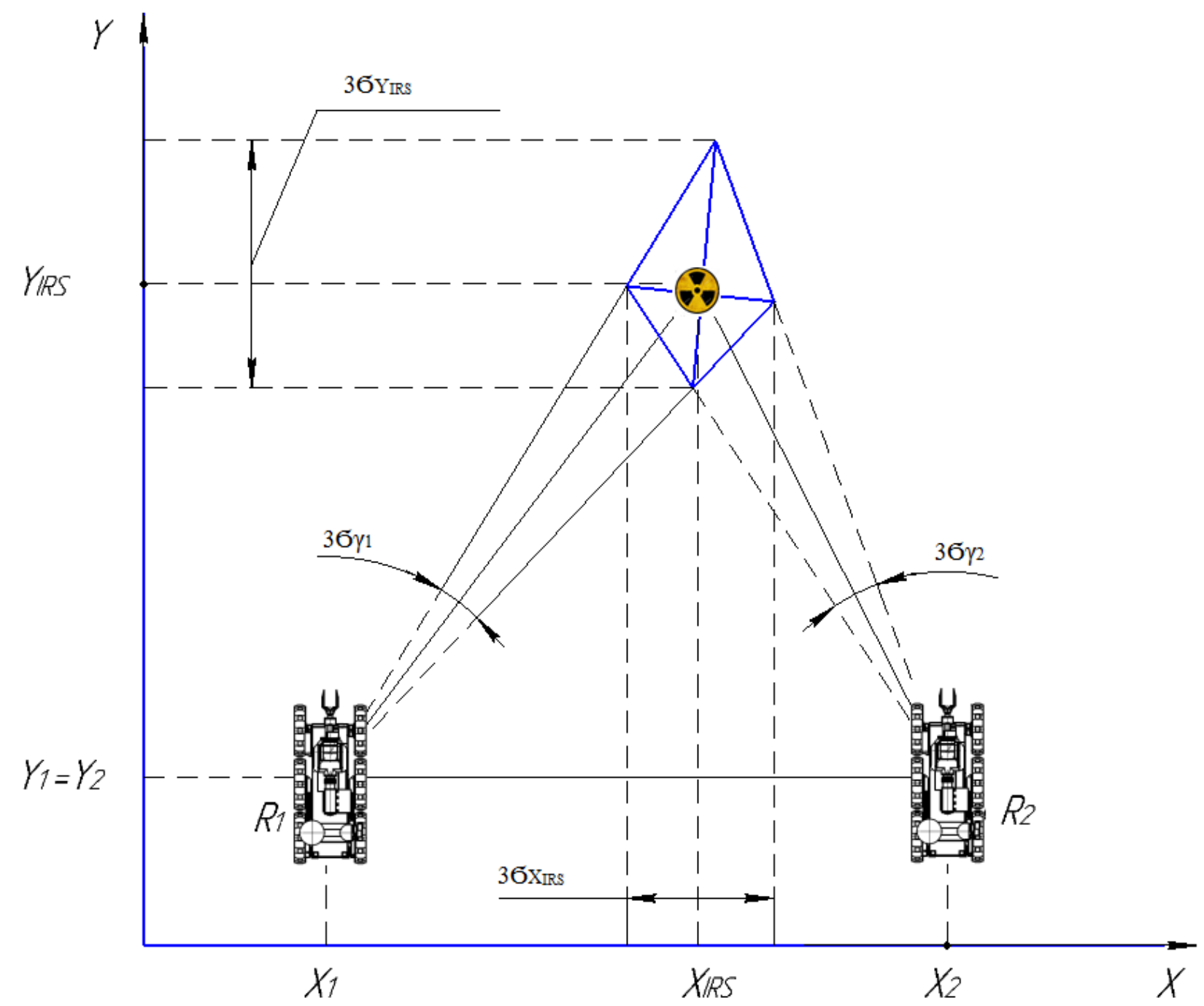

Fig. 4. IRS localization area

Results of calculations show that the minimum values of RMS for $\mathrm{X}_{\text {IRS }}$ and $\mathrm{Y}_{\mathrm{IRS}}$ functions can be achieved on condition that angles $\gamma_{1}$ and $\gamma_{2}$ are in the range of $32^{\circ}$ to $48^{\circ}$. Thus, the best initial positioning of robots is believed to be found.

For further simulation the following algorithm of search-mission organization has been chosen:

- estimating IRS angular directions by both robots simultaneously with accuracy error of 6;

- calculating of refined (in comparison to a priori) coordinates of IRS and its localization area (see Figure 4);

- non-stop moving of robot equipped with manipulator according to the shortest possible distance to the borders of

IRS localization area;

- defining refined direction to IRS and the refined IRS coordinates from calculated localization area border;

- entering into the IRS operation zone for robot equipped with manipulator in the way described for single robot.

The results of the search-mission simulation for options I-IV of initial conditions are presented in Table 3.

\begin{tabular}{|l|c|c|c|c|}
\hline \multicolumn{1}{|c|}{ Characteristics } & \multicolumn{4}{|c|}{ Value } \\
\cline { 2 - 5 } & \multicolumn{4}{|c|}{ Option } \\
\cline { 2 - 5 } & I & II & 1111 & 1165 \\
\hline $\begin{array}{l}\text { Average mission operation time for IRS } \\
\text { searching, s }\end{array}$ & 1645 & 2300 & 100 & 100 \\
\hline Successful missions percentage, \% & 100 & 100 & 100 \\
\hline
\end{tabular}

Table 3. Results of calculation of IRS search time for two robots operating in automatic mode 


\section{Conclusion}

In the frames of this work, disadvantages of existing methods and technologies for IRS search and localization have been defined. Analysis of current robotic complexes for radiation reconnaissance has proven robot group practicability application. Current technologies for IRS search and localization missions are not intended for use in group of robots. Defining the main procedures for operation fulfillment makes it possible to determine capabilities of improving to make them possible to be applied to heterogeneous robot group. Based on existing algorithms realized for search-mission application task by single robot, a number of mathematic simulation models have been made for heterogeneous robot group.

Simulation results show that time of operation for IRS search and localization task reduces by 2-5 times in correlation with initial conditions if a group of robots is applied. This means that integral absorbed radiation dose for every groupmember decreases as well. The percentage of successful missions in the case of robot group application reaches maximum of $100 \%$. Task-mission procedures described in this paper are meant for automatic mode operation to minimize participation of the human-operator in the mission, and this leads to efficiency effect increase [7]. On the basis of results of the research, robot group members are justified. Heterogeneous robot group should mainly include light class groundbased robot (up to $300 \mathrm{~kg}$ ) equipped with manipulator, ultra-light class ground-based robot (up to $50 \mathrm{~kg}$ ) and two smallsize helicopter type UAVs. Time of continuous operation for the members of such group corresponds to 2-3 hours, ensuring solution for the task of IRS search, localization and evacuation in correspondence with proposed technology. Simulation results also show that integral absorbed radiation dose is approximately by 1.4-2.0 times decrease (if compared to single robot operation process). When combined with corresponding services (decontamination, mitigation of consequences), there can be provided RC life and durability, speaking of both maintenance and midlife, full/complete and overhaul repairs. Dimensions and mass for both ground-based robots and UAVs make it possible to place them within single small-sized vehicle for transportation - for example, inside the cargo-passenger off-road vehicle [8]. Thus prompt response in conditions of emergencies can be granted.

The investigations held have proven the main disadvantage of aerial radiation reconnaissance - that is significant error of IRS localization due to field spatial smoothing. This means that elaboration of algorithms for data processing from UAV detection units, is necessary.

The results of work done will be further used in operating procedures for radiation reconnaissance complex being under development of RTC. They can also be applied for other robotic complexes and systems to solve detection and localization tasks.

\section{References}

[1] Yurevich E.I. Avariya na Chernobylskoi AES i ekstremalnaya robototekhnika [Chernobyl NPP Accident and Extreme Robotics]. Mechatronics, Automation, Control, 2004, vol.3, pp. 22-24 (in Russian).

[2] Boyko A.Yu., Vasiliev A.V., Denisienya Yu.A., Lipovckiy D.D. Opredeleniye prioritetnykh napravlenii razvitiya tekhnologii robototekchnicheskikh kompleksov voennogo naznacheniya voisk radiatsyonnoi, khimicheckoi I biologichesloi zashchity Vooruzhennykh Sil Rossiyskoi Federatsyi [Determination of the Priority Directions of Technology Development of Military Robotics of NBC Defense Troops of Russian Federation Armed Forces]. Robotics and Technical Cybernetics, 2017, vol.1(14), pp. 21-31 (in Russian)

[3] Kalyaev I. A., Gaiduk A. R., Kapustyan S. G. Modeli i algoritmy kollektivnogo upravleniya v gruppakh robotov [Models and Algorithms of Coordinated Control in Groups of Robots] Moscow, Fizmatlit, 2009, 280 p. (in Rssian)

[4] Russian State Scientific Center for Robotics and Technical Cybernetics. Available at: https://www.rtc.ru/ru/sredstva-radiatsionnogo-kontrolya/imd-24. (access date: 20.05.2017) (in Russian)

[5] Spassky B.A. Sovmestnoye primeneniye bespilotnykh apparatov razlichnogo bazirovaniya, Obzor sostoyaniya razvitiya [Application of Heterogenous Robotic Systems. State-of-the-Art]. Robotics and Technical Cybernetics, 2016, vol.2(11), pp. 8-19 (in Russian)

[6] Ventzel E. S., Ovcharov L. A. Teoriya veroyatnosti i yeyo inzhenernye prilozheniya [Probability Theory and Its Engineering Applications] Moscow, KNORUS, 2016. 480 p. (in Russian)

[7] Spassky B.A. Sovmestnoye upravleniye robotami, avtonomnoye i ot cheloveka-operatora [Robot Control: from Assisted Teleoperation and Mixed Initiative to Full Automation] Robotics and Technical Cybernetics, 2016, vol.2(11), pp. 69-76 (in Russian)

[8] Andreev V., Kirsanov K., Pryanichnikov V. (2016),Geographically distributed "multi-operator" Control for Mechatronic Devices via the Internet, In Proceedings of the 26th DAAAM International Symposium., B. Katalinic (Ed.), Published by DAAAM International, ISBN: 978-1-5108-1839-2, ISSN 1726-9679, Vienna, Austria. 2015. Curran Proceedings, New York,.pp. 0004-0011. 